\title{
A DUAL FORMULA FOR THE SPECTRAL DISTANCE IN NONCOMMUTATIVE GEOMETRY
}

\author{
FRANCESCO D'ANDREA, PIERRE MARTINETTI
}

\begin{abstract}
In noncommutative geometry, Connes's spectral distance is an extended metric on the state space of a $C^{*}$-algebra generalizing Kantorovich's dual formula of the Wasserstein distance of order 1 from optimal transport. It is expressed as a supremum. We present a dual formula - as an infimum - generalizing Beckmann's "dual of the dual" formulation of the Wasserstein distance. We then discuss some examples with matrix algebras, where such a dual formula may be useful to obtain upper bounds for the distance.
\end{abstract}

\section{INTRODUCTION}

Noncommutative geometry [2] extends Gelfand duality (that holds between locally compact Hausdorff spaces and commutative $\mathrm{C}^{*}$-algebras) to Riemannian geometry. In his reconstruction theorem [3], Connes has shown that all the geometric informations of an oriented closed Riemannian (spin) manifold $M$ are encoded within a suitable triple of algebraic data,

$$
A:=C^{\infty}(M), \quad H:=L^{2}(M, E), \quad D:=c \circ \nabla^{E}
$$

where $E \rightarrow M$ is an Hermitian vector bundle equipped with a unitary action $c$ of the Clifford algebra bundle and a connection $\nabla^{E}$ compatible with the Riemannian metric, and $C^{\infty}(M)$ is the (commutative) algebra of complex-valued smooth functions on $M$ that acts by pointwise multiplication on the Hilbert space $L^{2}(M, E)$ of square integrable sections of the bundle. Typically, if one is interested in the Riemannian structure only, then $E$ is the bundle of complex differential forms on $M$, with $D$ the Hodge-de Rham operator; or - if one is interested also in the spin structure in case $M$ is a spin manifold - then $E$ is the bundle of spinors with $D$ the associated Dirac operator (see e.g. [8]).

The triple (1) is the canonical example of a spectral triple, consisting in an associative — but not necessarily commutative - complex *-algebra $A$ acting faithfully on a Hilbert space $H$ through a bounded representation $\pi$, together with a selfadjoint operator $D$ on $H$ such that $[D, \pi(a)]$ is bounded and $\pi(a)\left(1+D^{2}\right)^{-1}$ is a compact operator for all $a \in A$.

2010 Mathematics Subject Classification. 58B34.

Key words and phrases. Spectral triples. Connes' distance. Beckmann's problem. 
The reconstruction theorem [3] shows that if a spectral triple $(A, H, D)$, with $A$ unital and commutative, satisfies some additional assumptions first listed in [4], then there exists a closed oriented Riemannian manifold $M$ such that $A=$ $C^{\infty}(M)$. A spin manifold is rebuilt if one adds more assumptions. Inspired by this geometric example, one refers to a spectral triple where $A$ is non-commutative as a noncommutative geometry.

In physics, there are strong theoretical motivations to believe that at small scale, the geometry of spacetime is not well captured by the notion of manifold. Geometry is expected to become "noncommutative" (or "quantum", in a more physical language) at very small scale. There exists many attempts to describe such "noncommutative spaces" in the mathematical physics literature (see e.g. [10] and reference within). But Connes' approach is the only one that provides a definition of a distance that coincides with the geodesic distance in the commutative case, and still makes sense in the noncommutative framework. ${ }^{1}$

Namely, given a spectral triple $(A, H, D)$, the spectral distance between any two states $\varphi_{1}, \varphi_{2}$ of $\bar{A}$ (the closure of $A$ with respect to the operator norm on $H$ ) is [5]:

$$
d_{D}\left(\varphi_{1}, \varphi_{2}\right):=\sup _{a \in A}\left\{\left|\varphi_{1}(a)-\varphi_{2}(a)\right|:\|[D, \pi(a)]\| \leq 1\right\} .
$$

It is not difficult to check that, for an arbitrary spectral triple, the formula (2) defines a distance (more precisely an extended metric, since (2) may be infinite) on the space $S(\bar{A})$ of states of $\bar{A}$. In the commutative case (1), the spectral distance (2) between pure states of the algebra $\bar{A}=C(M)$ of continuous functions on $M$, which are in 1-to-1 correspondence with the points of $M$, coincides with the geodesic distance. ${ }^{2}$ The meaning of the spectral distance between non-pure states, in the commutative case, was first noticed by Rieffel in [14]. He pointed out that (2) applied to (1) is nothing but Kantorovich dual formulation of the Wasserstein distance $W_{1}$ of order 1 studied in optimal transport, with cost function given by the geodesic distance $d_{\text {geo }}$ on $M$. States of $C(M)$ - that is positive, normalized, linear functionals $\varphi$ on $C(M)$ - are in 1-to-1 correspondence with probability measures $\mu$ on $M$,

$$
\varphi(f)=\int_{M} f d \mu \quad \forall f \in C(M)
$$

One then has

$$
d_{D}\left(\varphi_{1}, \varphi_{2}\right)=W_{1}\left(\mu_{1}, \mu_{2}\right)
$$

\footnotetext{
${ }^{1}$ Often, "noncommutative spaces" are intended as objects whose "coordinates do not commute". In the most elaborated of these models, one is able to define a length operator. However, the interpretation of the spectrum of this operator in terms of distances between some noncommutative equivalent of points is far from obvious [13].

${ }^{2}$ If $M$ is not connected, one adopts the convention that points in different connected components are at infinite geodesic distance.
} 
with

$$
W_{1}\left(\mu_{1}, \mu_{2}\right):=\inf _{\gamma} \int_{M \times M} d_{\text {geo }}(x, y) d \gamma(x, y),
$$

where the infimum is on all probability measures $\gamma$ on $M \times M$ with marginals the measures $\mu_{1}$ ands $\mu_{2}$ defining the states $\varphi_{1}, \varphi_{2}$ through (3). Later [6], we show that (4) actually holds even for non-compact complete manifolds, with $C^{\infty}(M)$ in (2) replaced by the algebra of compactly supported smooth functions on $M$, or of smooth Lipschitz functions on $M$ vanishing at infinity.

Connes' formula (2) thus provides a generalization of Kantorovich dual formula to the noncommutative framework. In the commutative case, (5) provides a "predual" formula. A natural question is whether there exists a similar formula in the noncommutative case, that is a noncommutative version of (5). In other terms, given an arbitrary spectral triple $(A, H, D)$, is there a way to express the spectral distance (2) as an infimum rather than a supremum?

The interest is not just theoretical. In most of the explicit computations of the spectral distance, one usually "guesses" a good upper bound, then shows this is saturated. The difficulty is that while any $a \in A$ satisfying the side inequality $\|[D, \pi(a)]\| \leq 1$ gives a lower bound for the spectral distance:

$$
d_{D}\left(\varphi_{1}, \varphi_{2}\right) \geq\left|\varphi_{1}(a)-\varphi_{2}(a)\right|,
$$

there is no easy way to obtain an upper bound. On the contrary, in the commutative case, any probability measure $\gamma$ on $M \times M$ with marginals $\mu_{1}$ and $\mu_{2}$ gives an upper bound for the Wasserstein distance:

$$
W_{1}\left(\mu_{1}, \mu_{2}\right) \leq \int_{M \times M} d_{\mathrm{geo}}(x, y) d \gamma(x, y) .
$$

Notice that if upper and lower bounds in (6) and (7) coincide, the distance is actually computed. What is missing in the non-commutative case is a formula expressing $d_{D}$ as an infimum, from which one could easily get upper bounds.

A first attempt in this direction starts with the following observation: on a manifold, the cost function is retrieved as the Wasserstein distance between pure states. On an arbitrary spectral triple, replacing states by probability measures on the space $\mathcal{P}(\bar{A})$ of pure states, a natural candidate for a pre-dual formula would be the Wasserstein distance $W_{D}$ on $\mathcal{S}(\bar{A})$, with cost the spectral distance on pure states (but note that the map from probability measures on $\mathcal{P}(\bar{A})$ to $\mathcal{S}(\bar{A})$ is surjective but, in general, not injective). One shows [11] that $d_{D}\left(\varphi_{1}, \varphi_{2}\right) \leq$ $W_{D}\left(\varphi_{1}, \varphi_{2}\right)$ for any states $\varphi_{1}, \varphi_{2}$, with equality when $\varphi_{1}$ and $\varphi_{2}$ are convex linear combinations of the same two pure states. However the equality does not hold in general (see the counterexample in [12]).

A similar approach is in [7, Sec. 4.6]: on the Berezin quantization $A$ of a compact homogeneous $G$-space $M$, with $G$ a connected compact semisimple Lie group, the 
symbol map associates to every quantum state a unique probability measure on $M$; one can then consider the Wasserstein distance between probability measures and show that it gives a distance on quantum states that is dual to a suitable seminorm on $A$, in the spirit of (2).

In this note, we show that there exists a dual formulation of the spectral distance (2) by adapting to noncommutative geometry the "dual of the dual" formula of the Wasserstein distance, also known as Beckmann's problem (cf. [18, Sec. 4.2]). This is inspired by the recent work [1] on matrix algebras. Our main result is Theorem 1 , which shows that the "dual of the dual" formula holds in full generality.

In the following, we will omit the representation symbol and identify an element of the algebra $A$ of a spectral triple with its representation as a bounded operator on $H$. By $A^{+}$we shall mean the subalgebra of $\mathcal{B}(H)$ generated by $A$ and 1 .

\section{A DUAL FORMUlation FOR THE SPECTRAL Distance}

Let $(A, H, D)$ be a spectral triple, and

$$
\Omega_{D}^{1}(A):=\operatorname{Span}\left\{a[D, b]: a, b \in A^{+}\right\}
$$

the $A$-bimodule of generalized 1 -forms. Denote by $\nabla$ the derivation:

$$
\nabla: A \rightarrow \Omega_{D}^{1}(A), \quad \nabla a:=[D, a] .
$$

By definition of a spectral triple, $[D, a]$ is bounded for any $a \in A$, so that $\Omega_{D}^{1}(A)$, and therefore $\operatorname{Im}(\nabla)$, is a subset of the algebra $\mathcal{B}(H)$ of bounded operators on $H$.

Let $B$ be any (complex) Banach subspace of $\mathcal{B}(H)$ containing $\operatorname{Im}(\nabla), B^{*}$ its Banach dual, i.e. the set of linear functionals $\Phi: B \rightarrow \mathbb{C}$ that are bounded for the operator norm

$$
\|\Phi\|:=\sup _{b \in B: b \neq 0} \frac{|\Phi(b)|}{\|b\|}
$$

and $\mathcal{L}(A, \mathbb{C})$ the set of linear maps $A \rightarrow \mathbb{C}$. We denote by

$$
\nabla^{*}: B^{*} \rightarrow \mathcal{L}(A, \mathbb{C})
$$

the pullback of $\nabla$ :

$$
\nabla^{*} \Phi(a):=\Phi(\nabla a), \quad \forall \Phi \in B^{*}, a \in A .
$$

Given a state $\varphi$ of $\bar{A}$ we will denote by $\varphi_{0}$ its restriction to $A$.

Theorem 1. Let $(A, H, D)$ be a spectral triple and, for any $\varphi, \psi \in S(\bar{A})$ at finite distance (i.e. $\left.d_{D}(\varphi, \psi)<\infty\right)$ and any Banach space $B$ containing $\operatorname{Im}(\nabla)$, define:

$$
W(\varphi, \psi):=\inf _{\Phi \in B^{*}}\left\{\|\Phi\|: \nabla^{*} \Phi=\varphi_{0}-\psi_{0}\right\} .
$$

Then: (i) the above inf is well-defined (the set of $\Phi$ satisfying the side condition is not empty), (ii) it is in fact a minimum and (iii) one has

$$
W(\varphi, \psi)=d_{D}(\varphi, \psi)
$$


In particular, (13) is independent of the choice of $B$.

Proof. Suppose $d_{D}(\varphi, \psi)<\infty$. Then $\varphi-\psi$ vanishes on ker $\nabla$. Indeed, let $b \in$ $\operatorname{ker} \nabla$. Set $a:=\lambda b$, with $\lambda \in \mathbb{C}$. Since $[D, a]=0$, one has

$$
d_{D}(\varphi, \psi) \geq|(\varphi-\psi)(a)|=|\lambda| \cdot|(\varphi-\psi)(b)| \forall \lambda \in \mathbb{C} .
$$

If $(\varphi-\psi)(b) \neq 0$, the sup over all $\lambda$ is infinite and we get a contradiction.

Let $B_{0}:=\operatorname{Im}(\nabla)$ and $\Phi_{0}: B_{0} \rightarrow \mathbb{C}$ be the map given by:

$$
\Phi_{0}(\nabla a):=\varphi_{0}(a)-\psi_{0}(a), \quad \forall a \in A .
$$

Since $\operatorname{ker} \nabla \subset \operatorname{ker}\left(\varphi_{0}-\psi_{0}\right)$, such a map is well defined. Note that

$$
\left\|\Phi_{0}\right\|:=\sup _{b \in B_{0}: b \neq 0} \frac{\left|\Phi_{0}(b)\right|}{\|b\|}=\sup _{a \in A:[D, a] \neq 0} \frac{|\varphi(a)-\psi(a)|}{\|[D, a]\|}=d_{D}(\varphi, \psi)
$$

is finite. By the Hahn-Banach theorem [17, Pag. 77, Cor. 1], the map $\Phi_{0}$ can be extended (non-uniquely) to a linear map in $B^{*}$ with the same norm, which proves the first statement.

Let $\Phi \in B^{*}$ be any of these extensions. Since by construction

$$
\nabla^{*} \Phi(a)=\Phi(\nabla a)=\Phi_{0}(\nabla a)=\varphi(a)-\psi(a) \quad \forall a \in A,
$$

we have from (16):

$$
W(\varphi, \psi) \leq\|\Phi\|=\left\|\Phi_{0}\right\|=d_{D}(\varphi, \psi) .
$$

On the other hand, for all $\Phi \in B^{*}$,

$$
\|\Phi\|=\sup _{b \in B: b \neq 0} \frac{|\Phi(b)|}{\|b\|} \geq \sup _{b \in B_{0}: b \neq 0} \frac{|\Phi(b)|}{\|b\|}=\sup _{a \in A: \nabla a \neq 0} \frac{\left|\nabla^{*} \Phi(a)\right|}{\|\nabla a\|} .
$$

In particular if $\nabla^{*} \Phi(a)=\varphi(a)-\psi(a)$, the right hand side of previous equation is $d_{D}(\varphi, \psi)$, which proves that $W(\varphi, \psi) \geq d_{D}(\varphi, \psi)$. The inf is attained on any extension $\Phi \in B^{*}$ of (15) with the same norm, thus it is a minimum.

Proposition 2. Let $(A, H, D)$ be a spectral triple and $\varphi, \psi \in S(\bar{A})$ two states with $d_{D}(\varphi, \psi)=\infty$. Then, there is no $\Phi \in B^{*}$ satisfying $\nabla^{*} \Phi=\varphi_{0}-\psi_{0}$.

Proof. Suppose $d_{D}(\varphi, \psi)=\infty$ and assume that there exists $\Phi \in B^{*}$ satisfying the side condition in (13). Then its restriction $\Phi_{0}$ to $\operatorname{Im}(\nabla)$ satisfies (15) and from (16) we deduce that $\Phi_{0}$ is unbounded, which is a contradiction since the norm of $\Phi_{0}$ should be bounded by the norm of $\Phi$.

Assumming by convention that the inf (13) on the empty set is $+\infty$, we have an equality between $W$ and $d_{D}$ for any pair of states.

It is worth noticing that, while (13) is always a minimum, the supremum in (2) is not always a maximum.

It is a maximum if the image of the set

$$
S:=\left\{a \in A^{+},\|[D, a]\| \leq 1\right\}
$$


under the quotient map $A^{+} \rightarrow A^{+} / \mathbb{C} 1$ is compact in the quotient topology induced by the norm topology on $A^{+}$. This happens in particular if $A$ is finite dimensional and $\operatorname{ker} \nabla=\mathbb{C} 1$ : the latter condition implies that $L(a):=\|[D, a]\|$ induces a norm on $A^{+} / \operatorname{ker} L=A^{+} / \mathbb{C} 1$, and $S / \mathbb{C} 1$ is the closed unit ball for this norm (which is compact since $A^{+} / \mathbb{C} 1$ is a finite-dimensional vector space). More generally, in the infinite-dimensional case and in the context of compact quantum metric spaces, the quotient unit ball is compact if $L$ is a closed Lip norm, cf. [15, Theorem 5.2]. Since, for all states $\varphi, \psi$, the difference $\varphi-\psi$ vanishes on constants as well, it defines a (continuous) function on $A^{+} / \mathbb{C} 1$. The spectral distance is the supremum of such a function on the closed unit ball $S / \mathbb{C} 1$, which is a maximum by Weierstrass theorem if the ball is compact.

As a corollary, if $S / \mathbb{C} 1$ is compact, then $d_{D}(\varphi, \psi)$ is finite for all $\varphi, \psi$ and the equality (14) holds for any pair of states.

\section{ExAmples}

3.1. Euclidean spaces. In this section we recover Beckmann's formula for the Wasserstein distance in $\mathbb{R}^{n}$ from formula (13). More precisely we show that the r.h.s. of the latter coincides with Beckmann's formula (given in (31) below).

Beckmann's formula deals with real valued functions, so first of all we need to check that the infimum in (13) can be equivalently searched on real valued $\Phi$. This follows from the observation [9] that the supremum in the distance formula (2) can be equivalently searched on the set $A^{\text {s.a. }}$ of selfadjoint elements of $A$, namely

$$
d_{D}\left(\varphi_{1}, \varphi_{2}\right)=\sup _{a \in A^{\text {s.a. }}}\left\{\varphi_{1}(a)-\varphi_{2}(a):\|[D, \pi(a)]\| \leq 1\right\} .
$$

Indeed, let $\nabla_{\mathbb{R}}$ be the restriction of $(9)$ to the real vector space $A^{\text {s.a. }}$. Take $B_{\mathbb{R}}$ to be any real Banach subspace of $\mathcal{B}(H)$ containing $\operatorname{Im}\left(\nabla_{\mathbb{R}}\right)$, denote by $B_{\mathbb{R}}^{*}$ its dual and $\nabla_{\mathbb{R}}^{*}: B_{\mathbb{R}}^{*} \rightarrow \mathcal{L}\left(A^{\text {s.a. }}, \mathbb{R}\right)$ the pullback of $\nabla_{\mathbb{R}}$. Then, starting from (20) and repeating almost verbatim the proof of the above theorems, using the HahnBanach theorem for real Banach spaces, one arrives at the following real version of Theorem 1.

Theorem 3. Let $(A, H, D)$ be a spectral triple and $\varphi, \psi$ two states such that $d_{D}(\varphi, \psi)<\infty$. Then

$$
d_{D}(\varphi, \psi)=\inf _{\Phi \in B_{\mathbb{R}}^{*}}\left\{\|\Phi\|: \nabla_{\mathbb{R}}^{*} \Phi=\varphi_{0}-\psi_{0}\right\}
$$

We now specialize to the canonical spectral triple of $M=\mathbb{R}^{n}$. In such a case, $H=L^{2}\left(\mathbb{R}^{n}\right) \otimes \mathbb{R}^{k}$, where $k=2^{[n / 2]}$ is the dimension of the spin representation, $D$ is the (flat) Dirac operator and $\nabla f=-i \sum_{\alpha=1}^{n} \gamma^{\alpha} \partial_{\alpha} f$ for all $f \in C_{c}^{\infty}\left(\mathbb{R}^{n}\right)$, with $\gamma^{1}, \ldots, \gamma^{n}$ Dirac's gamma matrices. Let $V \subset M_{k}(\mathbb{C})$ be the real vector subspace of complex $k \times k$ matrices spanned by $i \gamma^{1}, \ldots, i \gamma^{n}$, equipped with Hilbert-Schmidt 
inner product:

$$
\langle a, b\rangle_{\mathrm{HS}}:=\frac{1}{k} \operatorname{Tr}\left(a^{*} b\right), \quad \forall a, b \in M_{k}(\mathbb{C}) .
$$

With such a normalization, gamma matrices $i \gamma^{\alpha}$ form an orthonormal basis of $V$ :

$$
\left\langle i \gamma^{\alpha}, i \gamma^{\beta}\right\rangle_{\mathrm{HS}}=\frac{1}{k} \operatorname{Tr}\left(\gamma^{\alpha} \gamma^{\beta}\right)=\frac{1}{2 k} \operatorname{Tr}\left(\gamma^{\alpha} \gamma^{\beta}+\gamma^{\beta} \gamma^{\alpha}\right)=\frac{1}{k} \operatorname{Tr}\left(\delta^{\alpha \beta} \mathbb{I}\right)=\delta^{\alpha \beta},
$$

with $\mathbb{I}$ the identity matrix of dimension $k$. The image of $\nabla_{\mathbb{R}}$ is contained in the Banach space $B_{\mathbb{R}}:=C_{0}\left(\mathbb{R}^{n}, V\right)$ of continuous functions $\mathbb{R}^{n} \rightarrow V$ vanishing at infinity, with norm inherited from the operator norm on $H$. Any $b \in B_{\mathbb{R}}$ has the form $b=i \sum_{\alpha} f_{\alpha} \gamma^{\alpha}$, where $\boldsymbol{f}:=\left(f_{1}, \ldots, f_{n}\right)$ is an $n$-tuple of real-valued $C_{0}$-functions on $\mathbb{R}^{n}$. Since the operator norm is a $C^{*}$-norm and

$$
b^{*} b=\sum_{\alpha, \beta} f_{\alpha} f_{\beta} \gamma^{\alpha} \gamma^{\beta}=\frac{1}{2} \sum_{\alpha, \beta} f_{\alpha} f_{\beta}\left(\gamma^{\alpha} \gamma^{\beta}+\gamma^{\beta} \gamma^{\alpha}\right)=\sum_{\alpha}\left|f_{\alpha}\right|^{2} \mathbb{I}=|\boldsymbol{f}|^{2} \mathbb{I}
$$

the norm on $B_{\mathbb{R}}$ is just the Euclidean norm in $\mathbb{R}^{n}$ composed with the sup norm:

$$
\|b\|=\sup _{x \in \mathbb{R}^{n}}|\boldsymbol{f}(x)| .
$$

By Riesz Representation Theorem (see e.g. [16, Theorem 6.19]) the dual is $B_{\mathbb{R}}^{*}=$ $\mathfrak{M}\left(\mathbb{R}^{n}\right) \otimes V^{*}$, with $\mathfrak{M}\left(\mathbb{R}^{n}\right)$ the set of (real-valued) Radon measures on $\mathbb{R}^{n}$. Every $\Phi \in B_{\mathbb{R}}^{*}$ can then be expressed in the form

$$
\Phi(.)=i \sum_{\alpha} \int_{\mathbb{R}^{n}}\left\langle\gamma^{\alpha}, .\right\rangle_{\mathrm{HS}} d w_{\alpha}
$$

for some Radon measures $w_{1}, \ldots, w_{n}$. Given two states $\varphi, \psi$ corresponding to two measures $\mu, \nu$, the side condition $\nabla_{\mathbb{R}}^{*} \Phi=\varphi_{0}-\psi_{0}$ in (21) becomes:

$$
\int_{\mathbb{R}^{n}} \nabla f \cdot d \boldsymbol{w}=\int_{\mathbb{R}^{n}}(f d \mu-f d \nu), \quad \forall f \in C_{c}^{\infty}\left(\mathbb{R}^{n}\right),
$$

where $d \boldsymbol{w}=\left(d w_{1}, \ldots, d w_{n}\right), \boldsymbol{\nabla}=\left(\partial_{1}, \ldots, \partial_{n}\right)$ and "." is the standard Euclidean scalar product. In particular, if $d \boldsymbol{w}=\boldsymbol{w}(x) d^{n} x, d \mu=\mu(x) d^{n} x$ and $d \nu=\nu(x) d^{n} x$ for some $C^{1}$ functions, after integration by parts one gets:

$$
\int_{\mathbb{R}^{n}} f\{\boldsymbol{\nabla} \cdot \boldsymbol{w}+\mu-\nu\} d^{n} x=0 \quad \forall f \in C_{c}^{\infty}\left(\mathbb{R}^{n}\right) .
$$

Since $C_{c}^{\infty}\left(\mathbb{R}^{n}\right)$ is dense in $C_{0}\left(\mathbb{R}^{n}\right)$, the latter condition is equivalent to

$$
-\nabla \cdot \boldsymbol{w}=\mu-\nu .
$$

For more general Radon measures, the divergence condition (29) is to be read in the weak sense, i.e. as in (27).

The norm on $B_{\mathbb{R}}^{*}$, dual to $(25)$, is

$$
\|\Phi\|=\int_{\mathbb{R}^{n}}|\boldsymbol{w}(x)| d^{n} x .
$$


Combining (30) and (29), after a replacement $\boldsymbol{w} \rightarrow-\boldsymbol{w}$, Theorem 1 yields

$$
d_{D}(\varphi, \psi)=\min \left\{\int_{\mathbb{R}^{n}}|\boldsymbol{w}(x)| d^{n} x: \nabla \cdot \boldsymbol{w}=\mu-\nu\right\} .
$$

The r.h.s. of the above equation is precisely Beckmann's formula (4.4) in [18]. So we have recovered the later from the dual formulation of the spectral distance, as expected from Theorem 1.

Example 4. In simple cases, combining the formulas (31) and (2) one is able to explicitly compute the distance. For $n=1$, for example, the difference between the cumulative distributions

$$
w(x):=\int_{-\infty}^{x} d(\mu-\nu)
$$

satisfies the side condition in (31), hence we immediately get

$$
d_{D}(\varphi, \psi) \leq \int_{-\infty}^{+\infty}\left|\int_{-\infty}^{x} d(\mu-\nu)\right| d x
$$

We know on the other hand that the sup in (2) can be searched, in the commutative case, among the set of all 1-Lipschitz functions (see e.g. [6]). If $\mu-\nu$ has no singular continuous part, then (32) is piecewise continuous, its sign is piecewise continuous and the primitive of the sign is a 1-Lipschitz functions $\phi$. From (2) it follows that:

$$
d_{D}(\varphi, \psi) \geq\left|\int_{-\infty}^{+\infty} \phi d(\mu-\nu)\right|=\int_{-\infty}^{+\infty}\left|\int_{-\infty}^{x} d(\mu-\nu)\right| d x
$$

so that (33) is an equality.

3.2. Finite noncommutative spaces. In this section we show how to recover the "matricial distance" of [1] (more precisely their Theorem 1), which has been the motivating example of the present work. To this aim, we consider the spectral triple

$$
A=M_{n}(\mathbb{C}), \quad H=\mathbb{C}^{n} \otimes \mathbb{C}^{N}, \quad D=\sum_{i=1}^{N} L_{i} \otimes E_{i i}
$$

where $n, N \geq 1$, the algebra $A$ acts on the first factor of $H, L_{1}, \ldots, L_{N} \in M_{n}(\mathbb{C})$ are selfadjoint matrices and $E_{i j} \in M_{N}(\mathbb{C})$ is the matrix with 1 in position $(i, j)$ and zero everywhere else.

Recall that states $\varphi$ are in bijection with density matrices $\rho \in M_{n}(\mathbb{C})$, via the formula:

$$
\varphi(a)=\operatorname{Tr}(\rho a), \quad \forall a \in A .
$$

Choose the Banach space $B$ in (13) as follows:

$$
B=\underbrace{M_{n}(\mathbb{C}) \oplus \ldots \oplus M_{n}(\mathbb{C})}_{N \text { times }}
$$


where we think of elements of $B$ as block diagonal matrices with $N$ blocks of type $n \times n$. The formula

$$
\Phi=\sum_{i=1}^{N} \operatorname{Tr}_{\mathbb{C}^{n}}\left(u_{i} \cdot\right)
$$

gives a bijection between $u=\left(u_{1}, \ldots, u_{N}\right) \in B$ and $\Phi \in B^{*}$. From the cyclic property of the trace we deduce that

$$
\nabla^{*} \Phi(a)=\Phi([D, a])=\sum_{i=1}^{N} \operatorname{Tr}_{\mathbb{C}^{n}}\left(u_{i}\left[L_{i}, a\right]\right)=-\sum_{i=1}^{N} \operatorname{Tr}_{\mathbb{C}^{n}}\left(\left[L_{i}, u_{i}\right] a\right)
$$

for all $a \in A$, so that the side condition in (13) becomes

$$
-\sum_{i=1}^{N}\left[L_{i}, u_{i}\right]=\rho_{1}-\rho_{2}
$$

for two states with density matrices $\rho_{1}$ and $\rho_{2}$.

The dual of the operator norm in the finite-dimensional case is the nuclear norm $\|\cdot\|_{*}$ :

$$
\|\Phi\|=\|u\|_{*}:=\operatorname{Tr}\left(\sqrt{u^{*} u}\right) .
$$

Collecting all these informations, and since $u$ and $-u$ have the same norm, we arrive at the following theorem, specialization of our Theorem 1 to the present case.

Proposition 5. The spectral distance of the triple (35) is given by

$$
d_{D}\left(\varphi_{1}, \varphi_{2}\right)=\inf \left\{\|u\|_{*}: \sum_{i=1}^{N}\left[L_{i}, u_{i}\right]=\rho_{1}-\rho_{2}\right\},
$$

where $\varphi_{i}=\operatorname{Tr}\left(\rho_{i} \cdot\right), i=1,2$, are any two states at finite distance and the infimum is over all $u=\left(u_{1}, \ldots, u_{N}\right) \in M_{n}(\mathbb{C}) \oplus \ldots \oplus M_{n}(\mathbb{C})$ satisfying the side condition.

Using (21) instead of (13) one can show that the inf in (42) can be searched in the set of antisymmetric matrices, thus getting the same formula that is in [1].

We close this section with a simple (well-known) example where the sup and inf formula combined allow to easily compute the spectral distance.

Example 6. Consider the spectral triple (35) with $n=2, N=1$ (hence $H=\mathbb{C}^{2}$ ) and $D=L_{1}:=\left(\begin{array}{rr}1 & 0 \\ 0 & -1\end{array}\right)$. The correspondence

$$
\mathbb{R}^{3} \ni \vec{x}=\left(x_{1}, x_{2}, x_{3}\right) \mapsto \rho_{\vec{x}}:=\frac{1}{2}\left(\begin{array}{cc}
1+x_{3} & x_{1}-\mathrm{i} x_{2} \\
x_{1}+\mathrm{i} x_{2} & 1-x_{3}
\end{array}\right)
$$

maps the closed unit ball into density matrices. We will denote by $\varphi_{\vec{x}}$ the state with density matrix $\rho_{\vec{x}}$. 
Let $\vec{x} \neq \vec{y}$ be in the closed unit ball. Assume first that $x_{3}=y_{3}$. Define

$$
u=u_{1}:=\frac{1}{4}\left(\begin{array}{rr}
0 & \bar{z} \\
-z & 0
\end{array}\right), \quad a:=\frac{1}{2|z|}\left(\begin{array}{cc}
0 & \bar{z} \\
z & 0
\end{array}\right)
$$

where $z:=x_{1}-y_{1}+\mathrm{i}\left(x_{2}-y_{2}\right)$ and $z \neq 0($ since $\vec{x} \neq \vec{y})$. Both $u$ and a satisfy the side conditions in (2) and (42), so that

$$
\left|\varphi_{\vec{x}}(a)-\varphi_{\vec{y}}(a)\right| \leq d_{D}\left(\varphi_{\vec{x}}, \varphi_{\vec{y}}\right) \leq\|u\|_{*} .
$$

Since lower and upper bound are both equal to $\frac{1}{2}|z|$, the distance is computed:

$$
d_{D}\left(\varphi_{\vec{x}}, \varphi_{\vec{y}}\right)=\frac{1}{2}\|\vec{x}-\vec{y}\| .
$$

If $x_{3} \neq y_{3}$, on the other hand, one easily checks that the distance is infinite.

\section{REFERENCES}

[1] Y. Chen, T.T. Georgiou, L. Ning and A. Tannenbaum, Matricial Wasserstein-1 Distance, IEEE Control Systems Letters 1 (2017).

[2] A. Connes, Noncommutative geometry, Academic Press, 1994.

[3] A. Connes, On the spectral characterization of manifolds, J. Noncom. Geom. 7 (2013), no. 1, $1-82$.

[4] A. Connes, Gravity coupled with matter and the foundations of noncommutative geometry, Commun. Math. Phys. 182 (1996), 155-176.

[5] A. Connes and J. Lott, The metric aspect of noncommutative geometry, Nato ASI series B Physics 295 (1992), 53-93.

[6] F. D'Andrea and P. Martinetti, A view on optimal transport from noncommutative geometry, SIGMA 6 (2010), no. 057, 24 pages.

[7] F. D'Andrea, Pythagoras Theorem in Noncommutative Geometry, in "Noncommutative Geometry and Optimal Transport", Contemporary Mathematics 676 (AMS, 2016), pp. 175-210.

[8] J.M. Gracia-Bondía, J.C. Varilly and H. Figueroa, Elements of noncommutative geometry, Birkhäuser, 2001.

[9] B. Iochum, T. Krajewski, and P. Martinetti. Distances in finite spaces from noncommutative geometry, J. Geom. Phy. 31 (2001), 100-125.

[10] P. Martinetti, Line element in quantum gravity: the examples of dsr and noncommutative geometry, Int. J. Mod. Phys. A 24 (2009), no. 15, 2792-2801.

[11] P. Martinetti, Towards a Monge-Kantorovich distance in noncommutative geometry, Zap. Nauch. Semin. POMI 411 (2013).

[12] P. Martinetti, Connes distance and optimal transport, J. Phys. Conf. Series 968 (2018), 012007.

[13] P. Martinetti, F. Mercati, and L. Tomassini, Minimal length in quantum space and integrations of the line element in noncommutative geometry, Rev. Math. Phys. 24 (2012), no. 5, 1250010-36 pages.

[14] M.A. Rieffel, Metrics on states from actions of compact groups, Documenta Math.3 (1998), $215-229$

[15] M.A. Rieffel, Metric on state spaces, Documenta Math. 4 (1999), 559-600.

[16] W. Rudin, Real and complex analysis, McGraw-Hill, 1987.

[17] M. Reed and B. Simon, Methods of Modern Mathematical Physics I, Academic Press, 1980. 
[18] F. Santambrogio, Optimal Transport for Applied Mathematicians, PNLDE 87, Birkäuser (2015).

(F. D'Andrea) Università di Napoli "Federico II" and I.N.F.N. Sezione di Napoli, Complesso MSA, Via Cintia, 80126 Napoli, Italy

E-mail address: francesco.dandrea@unina.it

(P. Martinetti) Università di Genova (DIMA) and I.N.F.N. Sezione di Genova, via Dodecaneso 35, 16146 Genova, Italy

E-mail address: martinetti@dima.unige.it 